\title{
Consumo de drogas en adolescentes. Elementos de reflexión conceptual
}

\section{Adolescent Substance Abuse: Elements for a Conceptual Reflection}

\section{Abuso de substâncias por adolescentes: elementos para uma reflexão conceitual}

\section{Oscar Erazo Santander*}

Fecha de recepción: 25/09/2018

Fecha de evaluación: 29/10/2018

Fecha de aceptación: 05/11/2018

Disponible en línea:

DOI: https://doi.org/10.18359/reds.4357

Cómo citar este artículo:

Erazo, O. (2018). Consumo de drogas en adolescentes. Elementos de reflexión conceptual. Revista Educación y Desarrollo Social, 12(2), 15-31.

Psicólogo, especialista en intervención social y problemas humanos, máster en neuropsicología; cursos de doctorado en ciencias sociales, niñez y juventud. Es candidato a doctor en psicología con orientación en neurociencias cognitivas aplicadas. 


\title{
Resumen
}

El artículo tiene el objetivo de realizar reflexiones conceptuales sobre las drogas, consecuencias, intervención y vulnerabilidad en la adolescencia. En el primer apartado se explica el concepto de drogas y su prevalencia con resultados de más del $60 \%$ de consumidores adolescentes en Colombia. El segundo apartado nombra la acción de las drogas en el sistema dopaminérgico, la afectación en el sistema nervioso central, funciones ejecutivas y su logro en cuadros de dependencia y abuso; y analiza cómo los Estados intentan controlar la problemática con ofertas político-económicas de oferta y demanda. El tercer apartado explica la demanda a través de dos modelos: uno que muestra el consumo y consecuencias de tipo neurológico, y otro que describe los antecedentes para la decisión y acción de consumir, con resultados no concluyentes. Permite estructurar una propuesta hipotética que explica la elección y el consumo de drogas a través de un constructo teórico que integra una tesis de tipo cognitivo como la intencionalidad explicada por la teoría de conducta planificada, y la tesis neuropsicológica, explicada por las funciones ejecutivas con elementos como la toma de decisión, planeación e inhibición. El cuarto apartado refiere la tendencia del adolescente a escoger elecciones con desventaja y toma de decisiones inadecuadas con privilegio hacia el consumo, producto de la inmadurez en el funcionamiento ejecutivo, siendo este un elemento a intervenir.

Palabras clave: adolescentes y drogas; elección de consumo de drogas.

\begin{abstract}
This paper seeks to foster conceptual reflections on drugs, and their consequences, intervention, and vulnerabilities during adolescence. The first section describes the concept of drugs and their prevalence in consumption, since results evidence that more than $60 \%$ of users in Colombia are adolescents. The second section denotes the incidence of these substances on the dopaminergic system, their effects on the central nervous system and executive functions, and their role in cases of dependency and abuse. Moreover, this section assesses how governments are attempting to control this problem through political-economic supply and demand offers. The third section explains demand by means of two models, with the first one focusing on consumption and neurological consequences, while the second describes a background for consumption decisions and actions, offering non-conclusive results. Hence, within this context, a hypothetical proposal was structured to explain drug consumption choices through a theoretical construct that integrates a cognitive thesis. The fourth section refers to the propensity of adolescents to make poor choices, since the reported privilege toward consumption is a byproduct of immaturity, which is an element to intervene.
\end{abstract}

Keywords: drug use choices, adolescents and drugs.

\section{Resumo}

Este artigo procura promover reflexões conceituais sobre drogas e suas consequências, intervenções e vulnerabilidades durante a adolescência. A primeira seção descreve o conceito de drogas e sua prevalência no consumo, uma vez que os resultados evidenciam que mais de $60 \%$ dos usuários na Colômbia são adolescentes. A segunda seção denota a incidência dessas substâncias no sistema dopaminérgico, seus efeitos no sistema nervoso central, funções executivas e seu papel nos casos de dependência e abuso. Além disso, esta seção avalia como os governos estão tentando controlar esse problema por meio de políticas de oferta e demanda político-econômicas. A terceira seção explica a demanda por meio de dois modelos, com o primeiro enfocando o consumo e as consequências neurológicas, enquanto o segundo descreve um pano de fundo para decisões e ações de consumo, oferecendo resultados não conclusivos. Assim, dentro desse contexto, uma proposta hipotética foi estruturada para explicar as escolhas de consumo de drogas por meio de um construto teórico que integra uma tese cognitiva. A quarta seção refere-se à propensão dos adolescentes a fazer escolhas erradas, uma vez que o privilégio relatado em relação ao consumo é um subproduto da imaturidade, que é um elemento em que intervir.

Palavras-chave: escolhas pelo uso de drogas, adolescentes e drogas. 


\section{Drogas y consumo, prevalencia de una pandemia}

El consumo de drogas es considerado actualmente una pandemia, de difícil prevención e intervención y con posibilidad de generar patrones clínicos de dependencia y abuso que afectan la dimensión personal, social y económica de una sociedad (Becoña, 2002; Corominas, Roncero, Bruguera y Casas, 2007; Ministerio de Protección Social, 2008).

En 2015 existían 324 millones de consumidores, de los cuales 39 millones eran clasificados en dependencia (Echavarría y Giraldo, 2015; Pérez, Mejía, Reyes y Cardozo, 2015). En Suramérica la cantidad aumentó en 2010 al 60 \%, con preponderancia en adolescentes y necesitando de intervención clínica 2.4 millones de personas (Castaño y Uribe, 2014; Melo y Casthaneria, 2010; Toaza, 2011).

En Estados Unidos, 70.9 millones de personas son consumidores de nicotina, con inicio a los 12 años (National Institute on Drug Abuse, 2010a); en España, el $87 \%$ de sujetos entre 15 a 18 años fuma (Lloret, Morell-Gomis, Laguia y Moriano, 2017); y en el mundo 1200 millones de personas son fumadores con proyección a 2000 millones en 2030 (Becoña, 2002).

En Estados Unidos, 730.000 sujetos son adictos a los inhalantes con pico a los 14 años (NIDA, 2011); son consumidores de cannabis 19.8 millones, donde el 81 \% es adolescente (NIDA, 2015); existen
359.000 consumidores de crack; 11 millones de consumidores de éxtasis o MDMA (NIDA, 2007b; 2010b); y uno de cada seis estudiantes consume cocaína (Hidalgo y Redondo, 2005; NIDA, 2009).

En España, consume cannabis el 12 \% de los jóvenes entre 15 y 34 años, y uno de cada cuatro es adolescente (Lloret et al., 2017; Martínez y Ojeda, 2016); un 10 \% consume cocaína entre los 15 y los 18 años (Lloret et al., 2017); y un $6 \%$ consume éxtasis (Castellanos y Espinosa, 2013).

En Colombia, en 2013 se informaba que 480.000 menores ingresaban a urgencias con intoxicación por drogas y en 2015 un $63 \%$ de estudiantes de los grados 6 a 11 informan haber consumido alguna droga: tabaco el $24.3 \%$, marihuana el $7 \%$, cocaína el $2.8 \%$, basuco el $0.7 \%$, inhalantes el $3.1 \%$, éxtasis el $1.2 \%$, ácidos el $1.5 \%$, tranquilizantes el $1.1 \%$, popper el $2.5 \%$, heroína el $0.5 \%$ y dick el $4.1 \%$ (Andrade, Betancourt, Moreno y Alvis, 2017; Pérez et al., 2015). Son clasificados en dependencia de cannabis 250.000 sujetos, casi el $57 \%$ de consumidores de esta droga; de cocaína 140.000 , casi el $60 \%$ de consumidores de esta droga; y de basuco 34.000, siendo el $78 \%$ de consumidores de esta droga (Ministerio de Protección Social y Salud, 2008).

Para concluir, es identificable que la mayor prevalencia de consumo se presenta en la adolescencia, siendo la marihuana la droga más popular, seguida por la cocaína en América y Europa, y de opiáceos, de mayor uso en Asia con 
12.7 millones de consumidores (Echavarría y Giraldo, 2015). Pero también es frecuente el consumo de nuevas drogas como el kokodril y por dificultades de comercialización se han desplazado antiguas drogas populares por subproductos sintéticos como ha sucedido con la heroína por la morfina, fentanilo y desmorfina o el consumo de medicamentos con propiedades relajantes o activadoras (Castellanos y Espinosa, 2013).

El consumo actualmente es un fenómeno que se encuentra desbordado y sin capacidad de control. Pero, ¿cuáles son las consecuencias a nivel individual y social, que han llevado a los científicos de las ciencias de la salud y social a determinar su consumo como un problema o incluso una pandemia? Además, zpor qué su intervención justifica estrategias del Estado que pueden llegar a instancias militares? Cuestionamientos que se intentan resolver en los siguientes apartados a modo de reflexión.

\section{Drogas, consecuencias e inter- vención (un problema político)}

La droga es un concepto genérico, denominado así por su capacidad para generar respuestas con recompensa, producto de la activación biológica y neurológica del sistema nervioso central ante la sustancia (Organización Mundial de la Salud, 2005; Asociación Psiquiátrica Americana, 2014). Pero la sensación

18 de placer y bienestar tiene costos en la vida de los sujetos. Uno de ellos es la ausencia de control en el consumo.
La pérdida de control implica el pensamiento obsesivo y el comportamiento compulsivo por consumir la droga a pesar de sus consecuencias, hecho que no es producto de la función consciente, sino de la modificación biológica en el sistema nervioso central (SNC) y que se conoce como dependencia y abuso de sustancias psicoactivas (Alcázar, Verdejo, Bouso y Ortega, 2015; APA, 2014; NIDA, 2012; Martínez, 2012; Patiño, 2012; Sánchez, Arroyo y Azcona, 2014).

Con el consumo frecuente, los sistemas de comunicación caracterizados por la regulación de neurotransmisores y receptores operados molecularmente son modificados, llevándolos a su eliminación, inhibición o aumento (como sucede con receptores de dopamina y serotonina) (Quintero, 2015). La eliminación o inhibición de receptores termina por desconectar regiones cerebrales, como sucede con las conexiones entre el sistema límbico y la corteza prefrontal (CPF). Esta desconexión inactiva la CPF, entregando el control a la corteza subcortical encargada de mecanismos y comportamientos automáticos, reflejos y no conscientes (Ruiz et al., 2012).

La irregularidad en la CPF afecta el funcionamiento ejecutivo, describiendo deficiencias en la atención, memoria de trabajo, flexibilidad, planeación y resolución de problemas y los daños en la conexión subcortical llevan a desórdenes afectivos, conductuales y sociales caracterizados por depresión, tendencia suicida, dificultades al dormir, comportamiento impulsivo, hiperactivo, agresivo 
y tendencia disocial (Riaño, Guillen y Buela, 2015).

La alta estimulación de la droga en los centros de recompensa, sistema dopaminérgico o dopamina, lleva al desarrollo de conexiones que activan frecuentemente áreas subcorticales, como el núcleo accumbens, el tallo cerebral o los núcleos activadores de neurotransmisores (NT), relacionados con la sensación de placer, y los que no son controlados por la CPF. Su consecuencia se expresa en toma de decisiones impulsivas, desprovistas de juicio moral o riesgosas, como la sexualidad sin protección (vir, hepatitis C, embarazo no deseado) (Méndez, 2012; oms, 2005), conducción de automóviles (10\% de muertes relacionados con drogas) (Balogun, Koyanagi, Stickley, Gilmour y Shibuya, citado en Pérez et al., 2015) o la realización de conductas extremas de tipo negativista-desafiante o disocial, como lo ha identificado Pérez (citado en Fuenmayor y Saavedra, 2015) en una muestra de adolescentes con 1667 infractores y 9500 no infractores, en donde los infractores eran consumidores en el $75 \%$ de tranquilizantes, $50 \%$ de cocaína, $36 \%$ de inhalables, $21 \%$ de basuco, $12 \%$ de éxtasis y $6 \%$ de heroína.

Los cambios en el SNC generan tolerancia a la sustancia, caracterizada por la necesidad de incrementar la frecuencia y cantidad de droga para lograr un efecto de recompensa; la que en inicio fue de amplia magnitud, con el tiempo se transforma en experiencias desagradables y dolorosas (abstinencia). La compulsión y la necesidad de no sentir el dolor de la abstinencia llevan al sujeto a ingerir mayor cantidad, y un $6 \%$ de consumidores muere por sobredosis (NIDA, 2007a, 2010a).

Con el consumo también se dañan órganos internos como corazón, riñones e hígado, que terminan desarrollando cirrosis, deficiencias cardio-pulmonares, cáncer de pulmón, accidentes cerebrovasculares (NIDA, 2010a) y trastornos neurológicos y psiquiátricos (esquizofrenia, síndrome de korsakoff, psicosis, tendencia suicida, síndrome amotivacional) (Ministerio de Protección Social y Salud, 2008; Pérez et al., 2015).

El costo en Estados Unidos por consumo de tabaco y alcohol es de 10.7 millones de dólares y por drogas ilegales de 700.000 millones de dólares al año (López, 2012), sin contar las consecuencias sociales relacionadas, como la delincuencia, y de reducir o eliminar la actividad laboral y productiva de los Estados, los cuales pierden hasta 59 millones de dólares por consumo de tabaco, 58 millones por alcohol y 20 millones por drogas, condicionantes medidos para el año 2000 en Estados Unidos (Martínez, Pérez y De las Heras, 2008) con el índice AVAD (años de vida ajustados a discapacidad).

\section{Error en el modelo de intervención}

El innegable daño que producen las drogas, y su aumento en consumidores con consecuencias individuales, sociales, 
políticas y económicas, han llevado a los Estados a definir políticas para su intervención, las cuales se interpretan desde una visión económica y basada en preceptos de oferta y demanda. La tesis de la oferta sostiene que, al eliminar la producción, terminaría la problemática y, por el contrario, los teóricos de la demanda consideran que es necesario reducir la posibilidad de compra.

Ante la existencia de dos caminos para intervenir, Colombia, entre otros países, ha tomado la decisión de intervenir la oferta e incluir una política que elimine a productores y comerciantes, enfocándose en la tesis de que sin producto no hay oferta y sin oferta no hay demanda ni consumo (Thoumi, 2009). Uno de los ejemplos de esta propuesta es el Plan Colombia, desarrollado en convenio con los Estados Unidos y que hasta el año 2000 contó con recursos de 18.500 millones de dólares al año, más apoyo militar, permitiendo la creación de batallones especializados y la erradicación con glifosato. Entre sus resultados se cuentan miles de hectáreas destruidas y toneladas de droga incautada, encarcelamiento y muerte de carteles del narcotráfico ubicados en guerrillas, paramilitares y políticos del país, como lo afirman Morín y Collins (citados en Becoña, 2002; Pérez y Mejía, 2015).

Pero el Plan Colombia también llevó al país a una guerra civil, enfrentando a 20 narcotraficantes y militares de zonas urbanas y rurales por más de tres décadas, que como resultados llevo al aumento de la pobreza, el desplazamiento y el terro- rismo (Thoumi, 2009), y con el tiempo se considera un plan fallido, ya que en la actualidad solo es posible incautar una de diez drogas que se producen y sí, ha aumentado en el país consumidores y productores que involucran a nuevas mafias en todos los sectores, como el político, económico y judicial (Pérez y Mejía, 2015).

El error en la intervención de la oferta ha llevado a diversos países a repensar el paradigma y cambiar sus preceptos, que pasan de un concepto de represión a uno de legalización (Castaño y Uribe, 2014). En Colombia, el expresidente Juan Manuel Santos, en el marco de la cumbre de los Estados Latinoamericanos realizada en Cartagena en 2012, nombró la legalización de la marihuana (Pérez y Mejía, 2015) y diversos países del continente como Bolivia o algunos Estados de Estados Unidos han compartido estas consignas.

La idea de la posible legalización de las drogas, como ha sucedido con el alcohol y el tabaco, modifica el paradigma de observación del fenómeno, no enfocándose en la oferta, sino en el demandante o comprador. El comprador, que es el individuo que realiza la interacción, lo hace con elementos externalizantes (compra) pero también internalizantes (búsqueda y elección), convirtiendo al sujeto en responsable de la decisión y de mantener la oferta por su compra (Echavarría y Giraldo, 2015; Najul y Witzke, 2008; oms, 2011; Papazian, Alfonso y Luzondo, 2006; Portellano, 2005). 


\section{El demandante de una droga. Un problema de tipo cognitivo, neuropsicológico y del desarrollo}

Los estudios relacionados con las drogas y la conducta de consumo parecieran mostrar una línea divisoria y ubicarse en dos momentos específicos. Uno antes y otro después de consumir la primera droga. Los estudios enfocados en explicar qué sucede después de consumir la primera droga, han logrado profundizar sobre las consecuencias acontecidas en las estructuras neurológicas y neuropsicológicas provocadas por la toxicidad de la sustancia; además, han identificado el origen de los trastornos clínicos por el consumo, abuso y dependencia a través de la explicación basada en la perversión de los sistemas dopaminérgicos y su afectación en redes, conexiones con la corteza prefrontal y daños en las estructuras moleculares.

La segunda línea de investigación se centra en el antes de consumir, y basa sus preceptos en intentar comprender factores causales que llevan a la decisión de consumir. Entre sus conclusiones están las del modelo sistémico, que considera el acto de decidir y consumir como un hecho atravesado por diversos factores como el político, económico, social, familiar, afectivo, personal y biológico, todos en igual importancia y todos con posibilidades de afectación en la conducta. Una de sus conclusiones más preponderantes son los factores de riesgo y protección.
Los factores de riesgo y protección son un modelo que describe las relaciones existentes con las micro y macro estructuras del fenómeno, sin ánimo de ser concluyentes. Por ejemplo, se ha identificado que el consumo se asocia a condiciones de pobreza y ausencia de relaciones afectivas, sin embargo, existen sujetos con condiciones económicas y afectivas positivas también en consumo, con diferencias en la forma porcentual o el uso de una droga. Pero, finalmente, consumidores. Tal vez uno de los problemas que tiene este modelo se basa en un método que estudia relaciones entre procesos externos y consumo y no entre procesos subjetivos y consumo.

El factor subjetivo refiere a variables que describen las condiciones previas a la acción. Como lo propuesto por Ardila y Ostrosky (2008), quienes consideran que los individuos son capaces de realizar elecciones y conductas producto de sus representaciones y neuropsicología de la acción, y es esto es porque lo sujetos son poseedores de lenguajes interiores caracterizados por ideas, pensamientos y representaciones aprendidas en la interacción ambiental y el desarrollo de las funciones neurológicas del lenguaje.

El lenguaje interno, que en principio fue externo, se expresa a través de contenidos semánticos, sintácticos y pragmáticos, los cuales son utilizados como recursos e instrumentos manipulables por las funciones ejecutivas (FE). Estos que son producto de la corteza prefrontal y se desarrollan a través de los años y la madurez pero que sus formas de 
expresión dependen de la culturización y educación de los sujetos.

El lenguaje interno y su integración a las FE realizan acciones de planeación, memoria de trabajo, inhibición, toma de decisiones, entre otras, y construyen una diversidad compleja de mundos posibles con relación ante un fenómeno. Su resultado expresado en lenguaje interior y con nicho en la CPF se proyecta a través de redes hacia áreas sensorio-motrices en límites de la corteza frontal y parietal. La corteza sensorio-motriz primaria construye un mapa de acciones neurológicas que permite la activación del funcionamiento motriz y la secundaria realiza la acción, según lo definido por las estructuras mentales y neuropsicológicas (Martínez, Sánchez, Bechara y Roman, 2006; Sastre, Merino y Poch, 2007).

En conclusión, la elección y acción de una conducta son el producto de factores de tipo cognitivo expresados en representaciones, ideas o lenguajes internos, los que serían utilizados por las Fe para realizar toma de decisiones, planeación, inhibición o flexibilidad, procedimientos que suceden en la CPF y se relacionan con la integración de redes neurológicas del lenguaje y su expresión en productos de la CPF, que logran desarrollarse en comportamientos gracias al área sensorio-motriz que convierte las decisiones y su planeación en comportamientos con acción y movimiento.

Desde esta posición, la elección de consumir o inhibir una droga tiene relación con las representaciones mentales y su interacción con las FE, así los individuos tomarían la decisión con mayor ventaja y llegando a la realización de procesos de planeación, fluidez o inhibición de la conducta. Pero si bien la elección y la acción de consumo podrían tener relación con estos factores, se presentan nuevas preguntas reflexivas, como: ipor qué ante el estímulo (droga) los sujetos responden de forma diferente, algunos de forma positiva y otros negativa?, ¿unos inhiben y otros desarrollan acciones de consumo?, cuestionamientos que podrían responderse hipotéticamente desde las variables mencionadas.

\section{Elección de consumo de drogas, propuesta cognitiva y neuropsicológica}

En la psicología cognitiva, las representaciones mentales o también definidas como pensamientos o ideas, son de alta importancia, en tanto se consideran estructuras mentales con capacidad para inhibir, definir y desarrollar una conducta. Incluso para este paradigma la cognición y la conducta son sinónimos, al desarrollarse de forma continua y transversal. Así lo señalan algunos autores, como Bandura (aprendizaje vicario), Beck (ideas racionales-irracionales), Piaget (constructivismo y desarrollo del pensamiento) (Sánchez, 2009) y Vygotsky (pensamiento y lenguaje) (Martínez, Pérez y De las Heras, 2008; Torralba, 2015).

Dentro de este paradigma, emerge un concepto de importancia en la comprensión del antecedente comportamental y 
es la intencionalidad. La intencionalidad es una representación mental con contenido semántico que define y justifica la posibilidad o inhibición del desarrollo de una conducta o comportamiento y que, según Ajzen (citado por Martín, Martínez y Rojas, 2011) esta estructura mental también puede ser definida como creencia.

Las creencias son ideas con contenidos de favorabilidad o desfavorabilidad hacia un objeto o conducta, las cuales potencializan su posibilidad de acción. Según Ajzen (citado por Martín, Martínez y Rojas, 2011) estas creencias pueden ser de tres tipos: 1) creencias de actitudes con valoraciones positivas o negativas de un objeto o realidad, 2) creencias de norma subjetiva con percepciones positivas o negativas de otros respecto al objeto o conducta, y 3) creencias de control conductual percibido, sobre la existencia o deficiencia de recursos personales y ambientales para realizar una conducta. Según el autor, los análisis de las tres creencias definirían la intención de la conducta (Luengo, Romero, Gómez, Guerra y Lence, 2012; Peltzer et al., 2013).

El modelo con posibilidad de definir la intención predictiva hacia una conducta, ha sido denominado teoría de conducta planificada (TCP) (Ajzen citado por Martín, Martínez y Rojas, 2011) y goza de recomendaciones por la Organización Mundial de la Salud (oms) y la Organización Panamericana de la Salud (OPS) para su uso y con objetivos de prevención en estudios de consumo de drogas, sexuali- dad o prevención de enfermedades (oms, 2005; ops citado en Arbaiza, 2014; Tort, Cortes, Gimenez, Luque y Gomez, 2011; Peltzer et al., 2013; Lloret, Morell, Laguia y Moriano, 2017).

En el fenómeno de elección y consumo de drogas, la utilización de un modelo como la TCP permite describir los contenidos mentales sobre las drogas y además los tipos de creencias según actitud, norma subjetiva, control conductual e intencionalidad, que tienen los sujetos y que, según el modelo neuropsicológico, servirían de recursos para el procesamiento de las FE. Es necesario mencionar que los contenidos y complejidad de las creencias e intencionalidad no son similares en los sujetos, ya que estos dependen de los tipos de experiencias acontecidos en los sujetos (constructivismo).

Por su parte, las Fe son un factor de tipo neuropsicológico que explica cómo se desarrollan los procesos neurológicos y su funcionalidad a través de procesos superiores como la atención, la concentración, la memoria de trabajo (sostener, controlar y elegir información), la flexibilidad mental (Stelzer, Cervigni y Martino, 2010), la fluidez verbal (Tirapu, Muñoz y Pelegrin, 2002), la toma de decisión, la inhibición y la planeación.

Un ejemplo es la toma de decisiones (TD), que es un factor neuropsicológico resultado de la acción entre el sistema límbico y la CPF con interacción de NT como dopamina, serotonina y otros, representados en la acción para elegir una alternativa 
entre un rango de diversas propuestas (Bausela, 2007); su elección ofrecería una ventaja presente y futura (Verdejo, 2006; Oliva, 2007), pero su existencia no garantiza la elección de estímulos siempre con ventaja, ya que esta depende de procesos de madurez que involucran procesos biológicos y culturales.

Damazio y Bechara (citados en De Lorenzo, 2012) identificaron que los pacientes con daños neurológicos en la corteza orbito-frontal o sistema límbico describían la tendencia a realizar elecciones impulsivas, de riesgo, con desventaja o sin identificación de peligros. Decisiones y comportamientos frecuentes en sujetos con consumo de drogas (Donoghue y Rabin, 2000; Villalba y Verdejo, 2012) pero también en menor magnitud en adolescentes. La explicación para que los adolescentes tuvieran el comportamiento disfuncional se debe a que su cerebro a un esta inmaduro y se encuentra en un proceso de desarrollo consistente en la estructuración de redes y la organización de regiones orbito-frontal, ventro-medial y dorso-lateral que concluye en la segunda década de vida, pero que también se encuentra influenciado por los procesos culturales de educación y pautas de crianza (Asensio, 2011; Caña, Michilini, Acuña y Godoy, 2015).

La diferencia en la madurez de los sujetos debido a su desarrollo biológico y cambios en la estimulación cultural definiría las

24 diferencias en las formas de desarrollar el funcionamiento ejecutivo y sus productos, lo cual explicaría por qué las diferencias en las formas de elección y planeación ante un mismo estímulo, como lo es la droga entre los adolescentes.

Otras funciones ejecutivas implicadas son la planeación y la inhibición. La planeación se describe como la capacidad para generar programas meta de acción presente y futura y la inhibición como el proceso que permite controlar, regular e inhibir información sensorial, cognitiva o neurológica, que pudieran interferir el proceso. Las funciones de planeación e inhibición son las que, después de la decisión, estructuran un plan, bien sea para gestionar, buscar y desarrollar la acción de compra o para inhibirla.

Al igual que en la TD, la planeación y la inhibición en los seres humanos presentan formas diferentes de desarrollarse; esto no significa que no existan, pero su forma de modulación y ejecución se transforma, dependiendo de las condiciones biológicas y la madurez (Verdejo, 2006). Un ejemplo son los sujetos con trastorno por déficit de atención e hiperactividad, adicción, comportamiento impulsivo, negativista-desafiante o disocial, que puntúan en las pruebas de funcionamiento ejecutivo más bajo que los sujetos que no presentan estas deficiencias comportamentales (Flórez, Castillo y Jiménez, 2014; Flórez y Ostrosky, 2012).

Incluso los sujetos sin aparentes daños neurológicos, pero con trastornos de personalidad, bipolaridad, depresión o hijos de padres adictos, presentan diferencias en el funcionamiento ejecutivo, no mostrando patologías, pero sí deficiencias en el desarrollo ejecutivo 
(Rivarola, Pilatti, Godoy, Brussino y Pautassi, 2016). También en la adolescencia, y como producto de la inmadurez en el desarrollo de la CPF, los sujetos tendrían tendencia a mostrar deficiencias para la planeación e inhibición y otras funciones ejecutivas (Tirapu, Muñoz y Pelegrin, 2002; Verdejo y Pérez, 2005; Vivo et al., 2013), lo cual explicaría la heterogeneidad en la forma de realizar elecciones de consumo y su posterior acción.

\section{¿Por qué la elección de consumo de drogas en la adolescencia? Hipótesis para una explicación}

Los estudios que buscan definir los factores intervinientes en la decisión del consumo de una droga en la adolescencia son relevantes en tanto el $16 \%$ de sujetos inicia el consumo en esta etapa, e incluso la oms la ha definido como una enfermedad del desarrollo (NIDA, 2010b; Ministerio de Protección Social y Salud, 2008; Martínez y Ojeda, 2016; Méndez, 2012), nombrándose como un hecho preocupante, pues en 2009 en América Latina existían 150 millones de personas entre los 10 y 24 años; casi el $28 \%$ de la población, completamente vulnerables y en una época en la que no existe ni el conocimiento ni la política para prevenir o intervenir la pandemia con confiabilidad (Comisión Económica para América Latina y el Caribe; citado en Vivo et al., 2013).

Este es un hecho consecuente, en tanto para el adolescente esta etapa presenta cierta dificultad que no es controlable. Como lo es la activación hormonal, acompañada de transformaciones físicas, desarrollo sexual y deseo de una pareja, y cambios psicológicos que implican el camino hacia la independencia y la identidad, junto con el acoplamiento a estructuras y entramados sociales que exigen individualidad y al mismo tiempo una cohesión de grupo entre pares que excluye y distancian al sistema familiar.

Son múltiples los aspectos que debe resolver el adolescente, y que no cuenta con recursos suficientes de tipo neuropsicológico para permitirle una mejor coherencia en la toma de decisiones, planeación o inhibición de su intencionalidad. Estas condiciones lo hacen vulnerable ante un entramado biológico, psicológico y social que le exige una forma de interpretar y resolver la realidad, llenándolo de incertidumbre y conflicto (Luengo et al., 2012).

Pero el hecho de encontrarse en incertidumbre o conflicto no le impide tener la responsabilidad de tomar decisiones y realizar sistemas de planeación e inhibición. Tal vez el adolescente con contenidos de creencias e intencionalidad negativa hacia las drogas y el consumo y con positiva maduración de las Fe podría sortear la elección y lograr respuestas negativas ante el consumo, inhibiendo este comportamiento y activando otras estrategias de fluidez para una planeación diferencial.

Pero el adolescente con contenidos de creencias e intencionalidad positiva 
hacia las drogas e inmadurez en las FE posiblemente elegiría consumir una droga de forma impulsiva con recompensa actual, pero con desventaja futura (algunos reconocen las consecuencias, pero asumen la recompensa inmediata de mayor ventaja que la futura) organizando planes de corto y no de largo plazo, e inhibiendo acciones que no le permitan llegar a su cometido. En algunos casos el adolescente sin recursos neuropsicológicos no realizaría la elección tras su análisis respectivo, sino que, ante la presión por una decisión, terminaría realizando una elección promovida por el ambiente como pares, jíbaros o medios de comunicación y no por otros referentes como padres, ya que estos últimos ofrecen recompensas futuras, pero no inmediatas.

La elección positiva y la posterior gestión de consumo en el adolescente, en muchos casos, lleva a cuadros clínicos de dependencia y abuso. Algunos desertan de la escuela producto del bajo rendimiento o fracaso escolar, otros abandonan sus casas para continuar periodos profundos de consumo que se prolongan por periodos largos, acompañados de actividades delictivas y de calle (Cooke, Dahdah, Norman y French, 2016; Melo y Casthaneria, 2010; oms, 2005; Pérez y Mejía, 2015).

\section{Conclusiones}

26 El consumo de drogas y la adicción en la adolescencia son un fenómeno objetivo con una tasa de crecimiento superior al $60 \%$ en países latinoamericanos. Su acción y consecuencias le han llevado a definirse como una pandemia y enfermedad del desarrollo, y actualmente es de difícil promoción, prevención e intervención.

Las formas de intervención de tipo político y económico que se centran en la eliminación de la oferta y el control de la producción son, en su gran mayoría, ineficientes y se requiere de programas y políticas públicas con enfoque en el análisis y comprensión del fenómeno de la demanda y el sujeto comprador, las cuales expliquen las formas de decisión y acción de una conducta de consumo de drogas.

La elección y realización de conductas de consumo pueden ser explicadas desde tres variables de análisis. La primera, referida a factores cognitivos de intencionalidad y creencias, lo cual implica sus contenidos y complejidad; la segunda, los factores neuropsicológicos como las FE, las cuales utilizan las estructuras cognitivas para la toma de decisiones y posterior planeación, fluidez e inhibición; y, por último, está el factor de desarrollo y madurez, que explica que en la adolescencia los recursos neuropsicológicos aún están en desarrollo, y de ahí la alta vulnerabilidad a realizar elecciones impulsivas y con desventaja futura. 


\section{Referencias}

Ajzen, I. (2011). The theory of planned behaviour: Reactions and reflections. Psychology \& Health, 26(9), 1113-1127. DOI: https://doi.org/10.1080/0887044 6.2011 .613995

Alcazar, M., Verdejo, A., Bouzo, J. y Besos, N. (2010). Neuropsicología de la agresión impulsiva. Revista de neurología, 50(5), 291-299. Recuperado de https://www. neurologia.com/articulo/2009316

American Psychiatric Association (APA). (2014). Guía de consulta de los criterios diagnósticos del DSM-5. Washington, D. C.: American Psychiatric Publishing.

Andrade, P., Betancourt, D., Moreno, N. y Alvis, L. (2017). Fortalezas externas desde el modelo de desarrollo positivo de los jóvenes y consumo de sustancias en una muestra de adolescentes mexicanos y colombianos. Avances en Psicología Latinoamericana, 35(3), 515529. DOI:10.12804/a.4095

Arbaiza, M. (2014). Alteraciones cognitivas, conectividad funcional y personalidad en el drogodependiente. [Tesis para optaral título de Doctor en Neurociencias]. Madrid: Universidad Complutense de Madrid.

Ardila, A. y Ostrosky, F. (2008). Desarrollo histórico de las funciones ejecutivas. Revistade neuropsicología, neuropsiquiatría y neurociencias, 8(1), 1-21.

Asensio, S. (2011). Bases neuroanatómicas y neurofuncionales del trastorno de abuso de alcohol y su relación con la impulsividad: estudio mediante resonancia magnética. [Tesis para optar al título de Doctor en Fisiología]. Valencia: Universidad Cardenal Herrera.
Bausela, E. (2007). Implicaciones de las conexiones córtico y subcorticales del lóbulo frontal en la conducta humana. Revistaelectrónicade Motivación y Emoción, 10(25), 7. Disponible en http://reme. uji.es/articulos/numero25/revisio/texto

Becoña, E. (2002). Basescientíficas de la prevención de las drogodependencias. Ministerio del Interior. Delegación de Gobierno para el Plan Nacional sobre Drogas. Madrid, España. Recuperado de file:///C:/Users/ Win 10/Downloads/Bases\%20Cient_ ficas\%20Para\%20La\%20Prevencion\%20 De\%20Las\%20Drogodependencias\%20 -\%202002.pdf

Uribe, N. y Castaño, G. (2014). Barras de fútbol, consumo de drogas y violencia. Psicología desde el Caribe. Universidad del Norte, 31 (2), 243-279.

Caña, M., Michilini, Y., Acuña, G. y Godoy, J. (2015). Efectos de la impulsividad y el consumo de alcohol sobre la toma de decisiones en los adolescentes. Health and adicctions, 15(1), 55-66.

Castellanos, J.y Espinosa, G. (2013). Revisión de las tendencias de investigación sobre consumo de sustancias ilegales por los jóvenes. Virajes, 15(2), 57-71.

Corominas, M., Roncero, C., Bruguera, E. y Casas, M. (2007). Sistema dopaminérgico y adicciones. Revista Neurología, 44 (1), 23-31.

Cooke, R.; Dahdah, M.; Norman, P. y French, D. (2016). How well does the theory of planned behavior predict alcohol consumption? A systematic review and meta-analysis. Health Psychology Review, 10(2), 148-167. DOI: http://dx.doi.org/1 0.1080/17437199.2014.947547

De Lorenzo, D. (2012). La comprensión de las bases biológicas de la personalidad en el 
siglo XXI: genómica de la impulsividad En Celma, J. y Abella, F. (Edit.), Neuropsicología de laimpulsividad. Actualizaciones. España: Ediciones Universidad de Lleida.

Donoghue, T.y Rabin, M. (2000). The economics of immediaty gratificaction. Journal of behavioral decisión making. 13, 233-250. DOI: https://doi.org/10.1002/(SICI)1 099-0771(200004/06)13:2<233::AID -BDM325>3.0.CO;2-U

Echavarría, M. y Giraldo, A. (2015). Abordaje integral para la intervención de las conductas adictivas. Manual práctico para la detección, diagnóstico, remisión y tratamiento en el marco de la atención primaria en salud. Medellín: Secretaría de Salud de Medellín y Fundación Universitaria Luis Amigó.

Flórez, J., Castillo, R. y Jiménez, N. (2014) Desarrollo de las funciones ejecutivas, de la niñeza la juventud. Anales de psicología, 30(2), 463-473.

Flórez, J. y Ostrosky, F. (2012). Desarrollo neuropsicológico de lóbulos frontales y funciones ejecutivas. México: Editorial Manual Moderno.

Fuenmayor, D.y Saavedra, B. (2015). Sindrome disejecutivo en adolescentes en conflicto con ley penal. [Trabajo de investigación para optar al título de Profesional en Psicología]. Maracaibo, Venezuela: Escuela de Psicología, Universidad Rafael Urdaneta.

Hidalgo, M. y Redondo, A. (2005). Consumo de drogas en la adolescencia. Pediatric Integral, 9, 137-155.

Jiménez, C. (2013). Estudio de las funciones ejecutivas y la conducta social en jóvenes de 11 a 13 años. [Trabajo de grado para optar al título de Magíster en Neuropsicología Infantil]. Quito: Facultad de Ciencias
Psicológicas, Universidad Central del Ecuador

López, M. (2012). Determinantes de la conducta planeada y el significado del consumo de tabaco y/o alcohol y el consumo de estas sustancias en adolescentes. [Tesis para optar al título de Doctor en Ciencias de la Enfermería]. México: Facultad de Enfermería, Universidad de Nuevo León.

Luengo, M., Romero, E., Gómez, J., Guerra, A. y Lence, M. (2012). La prevención del consumo de drogas y la conducta antisocial en la escuela: análisis y evaluación de un programa. España: Universidad de Santiago de Compostela.

Lloret, D., Morell, R., Laguia, A. y Moriano, J. (2017). Diseño y validación de una escala de consumo de cannabis (Quic) para adolescentes. Adicciones, XX(x), 11-12.

Martínez, I. (2012). Efectos de la exposición y calidad de la prevención escolar en el consumo de drogas y factores de riesgo en estudiantes de secundaria. [Tesis doctoral]. Madrid: Facultad de Psicología, Universidad Complutense de Madrid.

Martín, M., Martínez, J. y Rojas, D. (2011). Teoría del comportamiento planificado y conducta sexual de riesgo en hombres homosexuales. Revista Panam Salud Pública. 29(6), 433-443.

Martínez, L. y Ojeda, T. (2016). Informe sobre hábitos de consumo de drogas entre adolescentes y jóvenes en espacios de ocio: resultados de intervención de una noche de fiesta. Unidad de prevención comunitaria en conductas adictivas. España: Ayuntamiento de Alfanfar.

Martínez, F., Pérez, E. y De las Heras, M. (2008). Nexus. Programaparalaprevención del consumo de drogas. Documento de 
trabajo no publicado. Deporte y vida Asociación deportiva. Madrid, España. Recuperado de http://www.fundacioncsz. org/ArchivosPublicaciones/166.pdf

Martínez, J., Sánchez, J., Bechara, J. y Roman, F. (2006). Mecanismos cerebrales de la toma de decisiones. Revista de neurología, 42(7), 411-418. Recuperado de https:// www.neurologia.com/articulo/2006161

Méndez, M. (2012). Variables de conducta, factores de riesgo para la salud y adaptación integral relacionados con la problemática bullying en estudiantes de educacion secundaria. [Tesis doctoral]. Murcia, España: Facultad de Psicología, Universidad de Murcia.

Melo, D. y Casthaneria, L. (2010). Autoeficacia y actitud: hacia el consumo de drogas en la infancia. Revista Latino-am Enfermagen, 18(spec)., 658-662.

Ministerio de Protección Social y Salud. (2008). La Política Nacional de Reducción del Consumo de spa al alcance de tod@s: Una herramienta para construir para socializar y construir política en lo social. Bogotá: Ministerio de Protección Social, Dirección General de Salud Pública. Recuperado de http://portalterritorial. gov.co/apc-aafiles/7515a587f637c2c66d$45 f 01 f 9 c 4 f 315 c / p o l t i c a-n a c i o n a l-d e-r e-$ ducci.pdf

National Institute on Drug Abuse (NIDA). (2007a). Abuso de los esteroides anabólicos. Serie de reportes de investigación. Recuperado de https://d14rmgtrwzf5a. cloudfront.net/sites/default/files/esteroides.pdf

National Institute on Drug Abuse (NIDA). (2007b). Abusoy adicción a la metanfetamina. Serie de reportes de investigación. Recuperado de https://dl4rmgtrwzf5a. cloudfront.net/sites/default/files/rrmetanfeta.pdf

National Institute on Drug Abuse (NIDA). (2010a). Adicción al tabaco. Serie de reportes de investigación. Recuperado dehttps://d14rmgtrwzf5a.cloudfront.net/ sites/default/files/rrtabaco_0.pdf

National Institute on Drug Abuse (NIDA). (2010b). Cocaína abuso y adicción. Serie de reportes de investigación. Recuperado dehttps://d14rmgtrwzf5a.cloudfront.net/ sites/default/files/rrcocaina.pdf

National Institute on Drug Abuse (NIDA). (2011). Abuso de inhalantes. Serie de reportes de investigación. National Institute on Drug Abuse. Advancing adicction science. Recuperado de https://www.drugabuse. gov/es/publicaciones/serie-de-reportes/ abuso-de-inhalantes/nota-de-la-directora

National Institute on Drug Abuse (NIDA). (2012). Los medicamentos de prescripción: abuso y adicción. Serie de reportes de investigación. Recuperado de https:// d14rmgtrwzf5a.cloudfront.net/sites/default/files/prescriptiondrugs_rrs_sp_1.pdf

National Institute on Drug Abuse (NIDA). (2015). La marihuana. Serie de reportes de investigación. Recuperado de https:// d14rmgtrwzf5a.cloudfront.net/sites/default/files/1832-la-marihuana.pdf

Najul, R. y Witzke. M. (2008). Funciones ejecutivas y desarrollo humano y comunitario. Kaleidoscopio, 5(9), 58-74.

Organización Mundial de la Salud (омs). (2005). Neurociencia del consumo y dependencia de sustancias psicoactivas. Washington: Organización Mundial de la Salud.

Oliva, A. (2007). Desarrollo cerebral y asunción de riesgos durante la adolescencia. Apuntes de psicología, 25(3), 239-254. 
Papazian, O., Alfonso, I. y Luzondo, R. (2006). Trastornos de las funciones ejecutivas. Revista de neurología, 42(3), s45-50.

Patiño, J. (2012). Patrones de consumo de cocaina en los jóvenes. [Tesis para optar al título de Doctor en Ciencias Sociales, de la Educación y la Salud]. España: Universidad de Girona.

Peltzer, R., Brandariz, R., Biscarra, M., Santángelo, P., Conde, K. y Cremonte, M. (2013). Predictores de la intención de consumo intensivo episódico de alcohol en adolescentes. Temas en psicología, 21(1), 183-192. DOI: 10.9788/TP2013.1-13

Pérez, A. y Mejía, J. (2015). Colombia, prevención del consumo de drogas y alcohol en Colombia. En Pérez, A., Mejía, J. y Becoña, E. (Eds.), De la prevención y otras historias. Historia y evolución de la prevención del consumo de alcohol y drogas en América Latina y en Europa (Capítulo 4). Recuperado de http://www.irefrea. eu/uploads/PDF/Perez-etal_Ed_Prevencion-y-otras-historias_2015.pdf

Pérez, A., Mejía, J., Reyes. M. y Cardozo, F. (2015). Consumo de alcohol en menores de 18 años en Colombia: 2015. Segundo estudio en siete capitales y dos municipios pequeños. Bogotá: Corporación Nuevo Rumbo. Recuperado de http://nuevosrumbos.org/wp-content/ uploads/2015/12/Consumo-de-Alcohol-en-menores-de-edad-en-Colombia-2015.pdf

Portellano, J. (2005). Cómo desarrollar lainteligencia. Entrenamiento neuropsicológico de la atención y funciones ejecutivas. Madrid: Editorial Somos Psicología

Quintero, M. (2015). La prevención de las adicciones en contextos familiares y escolares: análisis del programa de preven- ción de la asociación proyecto Hombre "Entre todos". [Tesis para optar al título de Doctor en Curriculum y Profesorado e Instituciones Educativas]. España: Universidad de Granada.

Ruiz, J., Pedrero, E., Lozoya, P., Llanero, M., Rojo, G. y Puerta, C. (2012). Inventario de síntomas prefrontales para la evaluación clínica de las adicciones en la vida diaria: proceso de creación y propiedades psicométricas. Revista de neurología, 54(11), 649-663.

Riaño-Hernández, D., Guillen, A. y Buela-Casal, G. (2015). Conceptualización y evaluación de la impulsividad en adolescentes: una revisión sistemática. Universitas Psychologica, 14(3), $1077-$ 1090. DOI: http://dx.doi.org/10.11144/ Javeriana.upsy14-3.ceia

Rivarola, G., Pilatti, A., Godoy, J., Brussino, S. y Pautassi, R. (2016). Modelo de predisposición adquirida para el uso de alcoholen adolescentes argentinos. Suma psicológica. 23, 116-124. DOI: http://dx. doi.org/10.1016/j.sumpsi.2016.03.002

Sánchez, L. (2009). Las percepciones sociales como determinantes del consumo de psicoestimulantes entre los jóvenes riojanos. Observatorio Riojano sobre Drogas. España: Gobierno de la Rioja.

Sánchez, T., Arroyo, A. y Azcona, A. (2014). Relación entre consumo de sustancias, búsqueda de sensaciones, impulsividad y síndrome disejecutivo en población universitaria. Reduca - serie congresos alumnos, 6(4), 312-321.

Sastre, S. (2006). Condiciones tempranas del desarrollo y el aprendizaje: el papel de las funciones ejecutivas. Revista de neurología, 46(2), s143-151. 
Stelzer, F., Cervigni, M. y Martino, P. (2010). Bases neurales del desarrollo de las funciones ejecutivas durante la infancia y la adolescencia. [Revisión]. Revista chilena de neuropsicología, 5(3), 176-184.

Thoumi, F.(2009). Unamiradaregional: laeconomía delas drogasen America Latina. Drogas ilegales: algunas verdades incomodad para Colombia. En Colectivo Maloka (Ed.), La economía de las drogas ilícitas. Escenarios de conflicto y derechos humanos (Capítulo II). Pp. 54-73. Barcelona: Generalitat de Cataluña Departamento de Interior, Relaciones Institucionales y Participación Oficina de Promoción de la Paz y de los Derechos Humanos.

Tirapu, J., Muñoz, J. y Pelegrin, C. (2002). Funciones ejecutivas. Necesidad de una integración conceptual. Revista de neurología, 34(7), 673-685

Torralba, E. (2015). Estudio psicosocial de las actividades de ocio en la adolescencia. [Tesis para optar al título de Doctor en Psicología]. La Mancha, España: Universidad de Castilla.

Toaza, A. (2011). Percepción de riesgos asociados al consumo de drogas y su influencia en estudiantes de octavo año de básica y primero de bachillerato de los colegios Padre Miguel Gamboa. [Trabajo de grado para optar al título de Psicólogo Clínico]. Quito: Facultad de Ciencias Psicológicas, Universidad Central del Ecuador.

Tort, B., Cortes, M., Giménez, J., Luque, L. y Gómez, R. (2011). Elaboración de un cuestionario basado en la teoría de conducta planificada de Ajzen para evaluar el consumo de alcohol en atrancón en adolescentes. Revista española de drogodependencia, 36(4), 403-412.
Verdejo,A.y Pérez, M. (2005). Neuropsicologíaen elámbito de las drogodependencias. Evaluación de las funciones ejecutivas. Revista de la Asociación Proyecto Hombre, 53, 39-43.

Vivo, S.; Saric, D.; Muñoz, R.; McCoy, S.; López, P. y Bautista, S. (2013). Guía para medir comportamiento de riesgo en jóvenes. Estados Unidos: Banco Interamericano de Desarrollo.

Verdejo, A. (2006). Funciones ejecutivas y toma de decisiones en drogodependientes: rendimiento neuropsicológico y funcionamiento cerebral. [Tesis doctoral del departamento de personalidad, evaluación y tratamiento psicológico]. España: Universidad de Granada.

Villalba, E. y Verdejo, A. (2012). Procesamiento emocional, intercepción y funciones ejecutivas en policonsumidores de drogas en tratamiento. Trastornos adictivos, 15, 10-20. 